\title{
Mesenchymal stromal cells with enhanced therapeutic properties
}

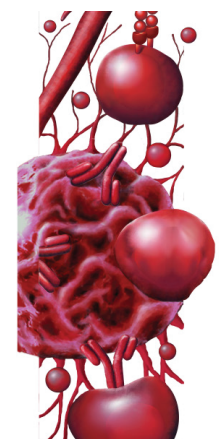

\begin{abstract}
Mesenchymal stromal cells (MSC) are connective tissue progenitor cells with interesting immunoregulatory properties and are currently being assessed as cellular therapeutics in a variety of clinical applications. While bone marrow has been the traditional source, adipose tissue and umbilical cord are being used increasingly to generate MSC for therapeutic use as an allogeneic, off-the-shelf product. Although the means by which MSC home to sites of inflammation or tissue damage and exert their beneficial effects remain to be fully elucidated, they have recently been shown to adsorb a number of immunosuppressive and anticancer drugs that may further enhance their therapeutic potential.
\end{abstract}

First draft submitted: 8 August 2016; Accepted for publication: 30 September 2016; Published online: 21 December 2016

Keywords: autoimmune disease $\bullet$ graft-versus-host disease $\bullet$ immunosuppressive drugs

- immunotherapy • rapamycin

\section{Background}

The functional definition of mesenchymal stromal cells (MSC) stipulates that they have the capacity to differentiate into osteoblasts, chondrocytes and adipocytes [1]. They also can develop into important stromal components of lymphoid tissues such as bone marrow (BM) and lymph nodes where they provide trophic support for hematopoietic stem cells and mature lymphocytes through provision of cytokines and growth factors [2-4]. In addition to the nurturing of immune cells, they have also been shown to inhibit the maturation, proliferation and function of most immune cell types [5-7]. It is these immunoregulatory properties that are being exploited in a large number of clinical trials, and most evidence now indicates that MSC promote tissue regeneration through inhibition of undesirable immune reactions and provision of growth factors rather than the direct replacement of damaged cells [8,9]. By contributing to an anti-inflammatory and nurturing environment, the infused MSC appear to allow for regeneration by endogenous cells, and they generally do not persist beyond days to weeks in the host.

Early-phase studies indicated tolerablity and preliminary evidence for efficacy when MSC were used to treat steroid-resistant acute graft-versus-host disease (GvHD) [10], multiple sclerosis (MS) and amyotrophic lateral sclerosis (ALS) [11], Crohn's fistula [12] and immune complications of solid organ transplantion [13]. The favorable indications from these early studies have led to a dramatic increase in activity and to date over 700 trials involving the application of MSC have been registered, with the large majority designed to test the ability of MSC to suppress unwanted immune reactions related to autoimmunity, or transplant-associated hostversus-graft reactions or GvHD (clinicaltrials.gov). Where the connective tissue credentials of MSC are invoked, such as in repair of wounds or fistulae, their immunoregulatory properties may also be playing important roles in promoting healing. Because of this
John Girdlestone

Stem Cells \& Immunotherapies Department, NHS Blood \& Transplant, Oxford, OX3 9BQ, UK

john.girdlestone@nhsbt.nhs.uk
Future $\because$ Medicine part of 
emphasis, this review will focus on the generation and enhancement of MSC for immunological applications. Genetic modification has the potential to selectively enhance function of MSC, but will not be covered here since it raises the cost and regulatory barriers to clinical use, and readers are referred to recent articles and reviews [14,15]. MSC-derived vesicles are an important new area of research and have been discussed elsewhere [16-18].

\section{Immunosuppression}

Extensive evidence has been provided for the suppression of a multitude of immune activities in vitro, and a number of enzymes and molecular messengers have been implicated in this immunoregulation $[5-7,19,20]$. Differences in the mechanisms employed by MSC of different species have been noted, with indoleamine 2,3-dioxygenase used by human cells to inhibit T-cell proliferation through the tyrosine depletion/kynurenine production [21], while murine MSC utilize inducible nitric oxide synthetase. Secreted mediators such as Prostaglandins and TSG- 6 as well as cell-cell contacts have also been shown to mediate immunosuppression. In order for MSC treatments to reach their full potential it is important that we develop a much greater understanding of how the cells exert their immunosuppression such that we can define release criteria [20], and determine which are the relevant pathways to enhance for optimal treatment of a particular clinical indication.

Despite the large numbers of preclinical studies and the thousands of patients who have been treated with MSC, evidence for their therapeutic efficacy remains suggestive rather than definitive. Relatively few trials reported to date have been randomized, controlled studies that were sufficiently powered to support robust conclusions, but systematic reviews are now being conducted to provide critical appraisals of clinical outcomes [23-26]. The trial limitations are partly a reflection of the young and rapidly expanding state of the field, and also due to the difficulty in recruiting and funding sufficiently large studies. The main conclusion to draw from clinical experience to date is that MSC appear to be safe for the patient at the commonly used doses of $1-10 \times 10^{6} / \mathrm{kg}$ [23]. However, full suppression of proliferation in vitro often requires up to one MSC per T cell, which will never be achieved by delivery in vivo. Questions have been raised as to how MSC might exert detectable effects at the low ratios with immune cells achieved upon infusion, and the prevailing answer is that MSC can localize to sites of inflammation or lymph nodes and exert their effects locally. Caution is warranted in that low ratios of MSC:target could potentially favor trophic rather than suppressive activities and therefore result in the survival of immune cells with undesirable activities, or tumorigenic cells [27,28]. Despite these caveats and a report of increased relapse rates in MSC-treated patients [29], suggesting a potential suppression of the graft-versus-leukemia effect, results to date have not indicated undue negative effects. However, the experimental nature of MSC treatments and the potential for unwanted skewing of the immune system warrants a vigilant monitoring of patients, as recommended by the International Society for Cellular Therapy (ISCT) [22] .

\section{Sources}

Autologous cells are preferable for avoiding immune barriers that may result in sensitization of the patient and/or destruction of the MSC, but the clinical need may not allow for the month or more required to obtain sufficient cells. The patient may be too ill to provide BM or adipose-derived MSC, or their cells may not possess the desired properties for congenital or disease-associated reasons (e.g., [30]). MSC derived from induced pluripotent stem cells would be another means to generate autologous cells under defined conditions for therapy, but again the time factor would limit the situations where they could be used [31-34]. In the setting of hematopoietic stem cell transplantation there can be the option of generating MSC from the original donor, in which case there is less risk of immune rejection. Although MSC can be generated pre-emptively from the donor before the stem cell harvest to avoid a delay in treatment in case GvHD develops, it can be difficult to justify the added medical intervention and cost of generating cells that may not be required.

Bartholomew et al. provided an early demonstration of immunosuppression by MSC with a primate skin graft model [35], and made the important observation that third party cells provided the same benefit as those from the skin donor. This opened the door to the use of allogeneic MSC, thereby transforming the logistics and cost calculations for their use as an off-the-shelf therapeutic product. MSC are routinely found to express low levels of HLA Class I proteins at their surface, and negligible amounts of class II or costimulatory signals [36]. While MSC are not invisible to the immune system, and can promote cytotoxic responses that may differ according to source or IFN- $\gamma$ pretreatment, they do not provoke strong allogeneic responses $[36,37]$.

The ability to use allogeneic cells without undue problems provides the opportunity to develop banks of MSC from a panel of unrelated donors that can be accessed at short notice. Production costs are reduced in comparison with bespoke MSC products, and the use of expanded cells from one or more donors can also 
allow for larger and/or repeated doses of MSC that may not be possible autologously, or from a single, designated donor. Repeated doses may increase the risk of developing anti-HLA antibodies or cytotoxic T lymphocytes directed against mismatched MSC, and the ISCT guidelines recommend monitoring of the development of anti-HLA responses [22]. Although the ideal MSC bank would provide for HLA-matching of donor to patient, a secondary option would be to use different donors for subsequent treatments, although this might only serve to broaden the patient's anti-HLA reactivity and complicate their treatment with other allogeneic products or organs. Serious complications arising from multiple dosings have not arisen as a major issue but it is unclear if some patients do not benefit because they rapidly remove MSC from circulation after subsequent infusions, as reported for mouse models [36,38]. If multiple doses of MSC are found to clinically useful but susceptible to neutralization by immune reactions, potential solutions would be to establish banks of HLA-typed MSC from donors, or derived from embryonic or induced pluripotent stem cells to cover the majority of potential patients [39].

$\mathrm{BM}$ has been the primary source of MSC for the majority of clinical applications, but there is increasing use of adipose-, placental-, and cord-derived MSC due to the relative ease of obtaining and expanding them $[40,41]$. The tissue source of allo-MSC may influence the degree of immune response, with adiposederived cells reported to stimulate a lower cytotoxic $\mathrm{T}$ lymphocyte response than those from BM [37]. MSC derived from specific organs may have distinct properties (e.g., liver [42]), but these are less likely to be used as banked products for immunotherapeutic use. The developmental capacities of MSC have been reported to differ by source [43-45] and, while this has fundamental implications for directed differentiation of MSC into specific cell types for tissue regeneration, we and others have not found significant differences in their immunosuppressive properties [43,46]. Preclinical engraftment studies indicated that cord and BM MSC were equivalent in promoting engraftment of $\mathrm{CD} 34^{+}$ hematopoeitic stem cells [47], but given the challenges of running randomized controlled trials it is unlikely that we will see head-to-head clinical comparisons of different MSC types within a reasonable time frame.

There is a limit to the amount of BM that can be provided by healthy donors or patients, while some adipose tissue donors can give more generously than others. The advantages of placental sources are the ability to acquire them in a noninvasive manner from a waste product, their ethical acceptability, and ready availability. The reported yields of MSC from cord vary dramatically from $10^{4}$ to $>10^{6}$ colony forming units per $\mathrm{cm}[48]$, indicating that isolation procedures require further investigation and optimization. GMP-compliant methods for dissociation of cord tissue to release MSC for expansion have been reported [49,50], and expansions on the order of $10^{5}$ are routinely reported. Given a global production of $130 \times 10^{6}$ babies per year, the associated $80,000 \mathrm{~km}$ of umbilical cord could be considered an inexhaustible source of MSC.

\section{Expansion}

The yield and potential for expansion are important considerations for choosing which source to use for therapeutic purposes, given the similarities between the immunoregulatory properties of MSC from different tissues when tested in vitro and in preclinical models [48,51]. Although many studies have used on the order of one to two doses of $1-2 \times 10^{6}$ cells per $\mathrm{kg}$, some recent trials have used almost ten-times this number [52], and some of the most promising results in the aGvHD field have been with pediatric patients where multiple doses (8-12) of $2 \times 10^{6} / \mathrm{kg}$ were applied [53]. Tenfold higher doses were used in the early skin graft model of Bartholomew et al. [35], but even these were only sufficient to delay rather than prevent rejection. The optimal MSC dosing regimens are not known since escalation studies are difficult and expensive to administer due to the logistics of assembling suitable patient cohorts, and to the challenges of generating sufficient cells within realistic budgets.

MSC are readily isolated from a variety of tissues, but in limited numbers such that expansion is required [48]. Adherence to tissue culture plastic has been the simple expedient for identifying and 'selecting' MSC, but specific markers have also been used to isolate specific subsets from heterogeneous MSC populations (e.g., STRO-1, CD271, CD146 [54-58]), further reducing the size of the primary cultures. While providing for a more defined product, it is important to demonstrate that such selections provide cells that display properties superior to those of the expanded starting population, rather than merely serving to distinguish them from those of a competitor. The main commercial approach has been to generate large numbers of cells from a small number of donors, thereby reducing costs associated with acquisition and testing. Although the production of large batches allows for consistency and comparisons across a number of patients, concerns have been raised as to whether the required population doublings might lead to reduced activity, senescence or karyotypic abnormalities [59]. Publicly funded studies have generally used cells at low passage numbers, with the consequent problem of batch-to-batch variability.

Fetal calf serum (FCS) has been used in the culture of MSC used for many trials to date, but there is 
increasing use of platelet lysate as an source of growth factors $[60,61]$. Although it provides a xeno-free alternative, precautions must be taken to ensure that platelet lysate does not transmit human pathogens [62]. It also is not a defined product and the essential components will need to be elucidated in order to provide for lotto-lot comparison. While totally defined, GMP-grade media are the ideal for expansion of MSC for clinical applications, cost considerations have been a barrier to their use for large-scale expansion to date. MSC expansion cultures continue to be carried out in multilayer flask systems but hollow fiber and microcarrier bioreactors are increasingly used to scale up production for clinical use [63-68].

Significant differences in the immunosuppressive properties of parallel samples expanded in flasks versus bioreactors have been reported [65], illustrating the need for potency standards to assist in optimizing production protocols (media compositions, degree of confluence attained, dissociation methods etc.) and defining release criteria $[9,22,65]$. A panel of surface markers provided the original criteria for MSC but did not serve to uniquely identify them, and the definition of their biological activity specified only trilineage differentiation and not their immunoregulatory activities [1]. The ISCT has recently released a paper addressing the issues of potency and other release criteria in light of regulatory body decisions on commercial MSC products, and discussing the matrix of assays that should be applied to product characterization [9]. These should include measurements of markers of known immunological function by flow cytometry, and analyses of mRNA and protein levels. The development of functional assays relevant to immunoregulation will be more challenging given the biological variability of responder cells, but the interesting use of lines with Treg properties can provide references for standardizing suppression assays [65].

\section{Enhancement}

Given the physiological and cost implications of expanding MSC sufficiently to provide larger and/ or multiple doses of MSC, an alternative approach is to boost their potency. By increasing the efficiency of their homing and immunoregulatory or other desired activities, it may be possible to infuse fewer cells or to reach clinical end points not achieved with current dosing regimens. MSC are thought to home to sites of tissue damage but only a very small proportion of those infused are likely to do so. By increasing the ability of MSC to localize to active sites of inflammation or tissue damage, and boosting their ability to provide relevant factors when they arrive, the efficacy of MSC treatments could be enhanced substantially.

\section{Homing}

Intravenous delivery has been the main mode of MSC delivery to patients, with the consequence that most of the cells are initially found in the lung, followed by distribution to the spleen and liver over hours to days [69,70]. The relatively large size of MSC in comparison with immune cell types may be the main reason for trapping in the lung, but receptor-mediated adhesion might also play a role. A number of groups are exploiting the deposition of MSC in the lung to treat indications such as COPD and ARDS [71]. While the cells are later found in other sites, the passive or active mechanisms by which the MSC localize are poorly understood. If sieving plays a major role, then it will be important to determine if selecting for smaller cells through the use of different sources or culturing techniques might promote redistribution. MSC-derived vesicles would not suffer the same size exclusion and it will be of interest to see if they can deliver tangible benefits to patients [16-18].

Once released from the lung, the objective is to promote homing to the affected tissues. The means by which MSC recognize and localize to such sites is unclear but may involve mechanisms similar to those used by leukocytes, such as integrin-mediated adhesion and chemotaxis toward cytokines [72-74]. MSC express a range of chemokine receptors with CXCR 4 being the most studied. The receptor for SDF-1/ CXCL12, CXCR4 has been shown to mediate chemotaxis by MSC [75], and its expression can be induced by a variety of culture conditions, cytokines and small molecule drugs $[76,77]$. However, many clinical applications of interest do not involve the CXCL12:CXCR4 axis and much needs to be done to understand how to direct MSC to skin, gut and so on [78] without altering their beneficial properties [79]. The development of methods for labeling MSC with clinically approved agents for tracking by high resolution imaging techniques in patients would contribute greatly to our understanding of the dynamics of infused cells $[80,81]$.

Where there are defined, limited sites of damage then direct injection of MSC into muscle or synovial joints has been employed [82], and arterial infusion has been used for treating acute kidney injury [83]. Encapsulated murine MSC-inhibited GvHD when injected subcutaneously [84], and encapsulated human MSC placed intraperitoneally inhibited inflammation and fibrosis in a murine model of liver disease [85]. These results indicate that systemic effects can be generated with localized cells, suggesting that production of soluble factors rather than homing and cell-cell contacts may be sufficient for therapy. Encapsulation prevents recognition of the MSC by host immune cells and thus may prolong their survival and extend the window of therapeutic activity. 
Licensing \& programming

Regulatory mechanisms by their nature should be responsive rather than constitutive and a constant, maximal immunosuppression exerted by MSC would not be conducive to the clearance of pathogens. Krampera and others have described a process of licensing, whereby MSC must be triggered by cytokines such as IFN- $\gamma$ or TNF- $\beta$ in order to exhibit potent immunoregulatory activities $[86,87]$. This situation is analogous to the negative feedback loop involving Treg which are sustained by IL-2 produced by activated effector cells. The ideal situation would be to inject naive MSC that would home to sites of inflammation and be instructed by the local milieu, but several arguments have been made for licensing MSC before infusion into patients: there may be insufficient IFN- $\gamma$ or other cytokines produced at sites of inflammation; there may be too short a time for licensing before they are cleared away; or the MSC may be converted into an unwanted state [87].

Evidence that MSC have the potential to respond to distinct pathogen associated molecular patterns is provided by their expression of Toll receptors [88,89]. This family of pattern recognition receptors is capable of binding pathogen associated molecular patterns and transmitting signals to the nucleus that culminate in the execution of programs of immune gene expression. Although a number of groups have seen distinct responses of MSC to ligands for TLR3 (double stranded viral RNA) and TL4 (bacterial cell wall components), there are divergent reports of the consequences for their immunosuppressive activities. While some saw no effect, others reported seeing reduction or enhancement by pretreatment with ligands for either TLR3 or -4 [90,91]. Waterman et al. [92] described the loss of immunosuppression by MSC primed with TLR-4 ligand, leading them to propose a paradigm of type I (pro-inflammatory) and II (anti-inflammatory) MSC, analogous to that described for monocytes, and they advocate skewing MSC before infusion in order to avoid them inhibiting desired immune actions [93]. The tailoring of MSC to exert appropriate activities is an important aim, but more work is needed to understand how to license MSC reproducibly and to identify protocols that will produce desired outcomes in vivo.

\section{Loading MSC with small-molecule drugs Immunosuppressive drugs}

MSC have been demonstrated to inhibit the activities of multiple cell types, and much remains to be determined regarding their involvement in immune regulatory networks. While investigating the MSC:monocyte interactions that are required for inhibition of T-cell proliferation [94], a number of cytokines and small molecules were tested for their ability to enhance or inhibit this communication. While pre-incubation of MSC with IFN- $\gamma$ or other cytokines tested showed little effect on their ability to inhibit proliferation of polyclonally stimulated $\mathrm{T}$ cells, rapamycin (sirolimus) substantially increased the potency of the MSC [95]. Although the original hypothesis was that inhibition of the mTOR pathway was leading to changes in the physiology of MSC that increased their immunosuppressive potency, subsequent experiments indicated that the main enhancement was due to release of rapamycin that had been adsorbed during pretreatment of the MSC. Starting with 48-h incubations, it was found that reducing drug exposure times to as short as $5 \mathrm{~min}$ were sufficient to produce a maximal enhancement. It is conceivable that significant metabolic changes might result from such brief treatments, but the observation led us to consider the possibility that the drug itself was causing the enhanced inhibition of T-cell proliferation. Indeed, even after trypsinization and thorough washing, rapamycin was found in the cell pellet at levels that were sufficient to inhibit $\mathrm{T}$ cells in our assays, assuming that the drug was capable of diffusing out from the MSC into the co-cultures. The observation that a neutralizing antirapamycin serum totally blocked the enhancement indicated that the MSC and the adsorbed drug were acting additively and independently to inhibit T-cell proliferation. When rapamycin was added at the same time as MSC to the assays, as opposed to use as a pretreatment, we observed a slightly less than additive effect. This had been reported by others [96], and we ascribe the decrease to a sequestration of the drug by the much larger MSC, such that the T cells are exposed to lower concentrations.

Rapamycin is hydrophobic, a property that complicates its delivery and leads to its partition into the cellular components of blood [97]. Our hypothesis is that when added to MSC cultures the drug is being adsorbed by the cells, but it is not known whether this is into lipid membranes and/or other compartments. The newer generation rapalogue everolimus is less hydrophobic than rapamycin but still distributes preferentially into the cellular fraction of blood [98], and it was found to enhance the immunosuppressive potency of MSC when used in pretreatments at similar concentrations to those for rapamycin. Since these two drugs are thought to have the similar mechanisms of action, it could still be that inhibition of the mTOR pathway is responsible for increasing the immunosuppressive potential of the MSC. However, pre-treatment with FK-506 (tacrolimus) at similar concentrations was found to provide an equivalent enhancement of MSC [95]. Although it binds to the same target (FKBP12) as rapamycin, it inhibits the calcineurin 
rather than mTOR pathway. Cyclosporin A (CsA) inhibits calcineurin through binding of a distinct immunophilin, but was also found to enhance MSC potency. Pretreatment with higher concentrations of CsA was required to achieve an effect, and this could be due to reduced adsorption by the MSC and/or the higher concentrations required to inhibit T-cell proliferation. Pretreatment of MSC with smaller, less hydrophobic drugs (azathioprine and mycophenolate) had no effects, indicating that not all immunosuppressive drugs (ISDs) are capable of enhancing potency.

If rapamycin partitions into MSC on a biophysical basis then other cell types should also adsorb the drug, and indeed immunosuppression by primary human fibroblasts and mouse embryo fibroblasts was substantially increased after brief pretreatments [95]. A fibroblast line and endothelial cells stimulated T-cell proliferation at lower ratios, but became suppressive after pretreatment. Interestingly, antigen-presenting cell preparations lost their allostimulatory properties after incubation with rapamycin, but a full mixedlymphocyte response was recovered if antirapamycin was included in the cocultures; therefore, the ability to adsorb rapamycin appears to be a general property of cells and is not unique to MSC. One can envision the benefits of using of organ or stem cell grafts that have been treated with ISD in order to reduce immediate graft-versus-host and host-versus-graft reactions.

Orange et al. [99] reported that dendritic cells pretreated with FK506 could trigger T-cell apoptosis through inhibition of IL-2 synthesis, and provided evidence that this was likely due to the adsorption and release of the drug. They exploited this activity to develop a system for antigen-specific killing of $\mathrm{T}$ cells and demonstrated an inhibition of collagen-induced arthritis in a preclinical model by the use of FK506treated DC pulsed with collagen II. Rapamycin antagonized the action of FK506 in their in vitro assay for IL-2 induction, ascribed to the competitive binding for FKBP12, but it would be of interest to see if rapamycin-treated DC can induce anergy or other modulations of T-cell activity.

The immunosuppressive potency of Treg has been reported to increase after incubation with rapamycin [100]. The drug is used to reduce residual $\mathrm{T}$ effector cell numbers in Treg expansion cultures since they are preferentially sensitive to its antiproliferative effects, although there conceivably could be an effect of prolonged mTOR inhibition on Treg functionality. However, we have found that $15 \mathrm{~min}$ treatments of Treg isolated from human adult or cord blood are sufficient to increase their potency [SRIVASTAVA S, PERS. COMM.]. Therefore, we suggest that the Treg are also adsorbing the rapamycin and acting as drug delivery vectors. As with MSC, the immunosuppression mediated by the treated cells will be the result of the combined actions of the native cellular mechanisms and release of the adsorbed drug.

If hydrophobic drugs are taken up rapidly by MSC and other cell types, then they presumably will be released down a concentration gradient when the cells are transferred to fresh medium without drug, or infused into patients. From simple wash-out experiments we estimated that the half-life of the drug-enhanced effect was about 1 day [95], but this would likely be shorter under repeated changes of surrounding solutions. Although MSC may only act over a few days when infused, it was unclear if the enhancement seen in vitro would persist long enough to exert a therapeutic effect. However, in a xenogeneic model of GvHD we found that a low dose of rapamycin-treated umbilical cord MSC was superior to fourfold more untreated cells, or rapamycin alone at much higher concentrations [95]. We calculated that about $50 \mathrm{ng}$ of drug would have been present in the infusion of treated MSC, yet $3 \times 50 \mu \mathrm{g}$ injections of were insufficient to inhibit disease symptoms. This indicates a strong synergy that was not observed in vitro, but in that situation all the components are placed in a culture well and there is no migration necessary for the actors to find themselves in close proximity. Sensitive tracking studies would be required for confirmation, but our interpretation of the pronounced increase in efficacy of the treated MSC seen in the GvHD model was that the cells localized to sites of inflammation where they utilized their arsenal of immunosuppressive agents, and in addition exuded rapamycin to inhibit immune activities. While one dose of treated MSC caused a significant delay in the onset of GvHD it did not totally suppress disease and future work will be needed to determine if larger and/or repeated doses can provide improved or complete protection. Due to animal welfare restrictions it was not possible to test whether the treated MSC were effective at treating ongoing GvHD, but this is a clinically important demand and it will be important to test in future. Kim et al. have also found that rapamycintreated human adipose MSC were better than untreated cells at suppressing a mismatched mouse model of GvHD [100].

Synergistic effects have been reported with the infusion of MSC together with systemic administration of rapamycin in models of heart [96] and pancreatic islet [103] transplantation, and the treatment of experimental autoimmune encephalomyelitis [104]. This indicates that they are acting through complementary mechanisms, consistent with our results. While it is too much of a leap to suggest that pretreated MSC, or other cells with homing potential such as Treg, will obviate the need for injection with ISD, they may well 
reduce the dosage requirements. By localizing the drug to sites of inflammation rather than exposing the whole body it may be possible to reduce the negative sideeffects of drugs, such as inhibition of wound repair by rapamycin that precludes its use in the period after transplantation during which it might exert beneficial immune effects [105]. It will be of interest to see if ISDtreated MSC are less likely to stimulate the production of donor-specific antibodies that have the potential to reduce effectiveness of multiply infused lots of MSC.

\section{Anticancer \& other drugs}

Pessina and colleagues have published an interesting body of work demonstrating that human MSC and mouse stromal cells are capable of taking up chemotherapeutic drugs such as paclitaxel [106] and gemcitabine [107], and the antibiotic ciprofloxacine [108]. The cells were relatively resistant to the effects of the drugs such that they could be used as vectors to deliver sufficient amounts to affect target cells. The loaded MSC were able to inhibit angiogenesis and proliferation of tumor cells in vitro, and also showed enhanced suppression of T-cell proliferation [109]. In animal models the drug-primed MSC were able to reduce tumor formation when co-injected with transformed cells and, encouragingly for therapeutic use, they reduced metastases produced from cells injected at earlier time points [110]. As we found with rapamycin, there was a synergistic effect that was greater than that achievable with treatment with drug or cells alone, indicating that focal delivery by the MSC was relevant.
Pessina et al. [110] note that 2000-fold less drug was delivered by the loaded MSC than would be used systemically, similar to the order of magnitude reduction associated with the rapamycin-treated cells used in our GvHD model. Although much work will be needed to determine if loaded MSC will work sufficiently well in clinical situations to allow dispensing with the systemic infusion of drugs, they may aid in treatment with drugs that are difficult to administer and/or where off-target effects reduce their therapeutic window.

Just as we found that MSC were not unique in their ability to adsorb rapamycin, the paclitaxel loading effect was seen with dermal fibroblasts [111], and with $\mathrm{CD} 14^{+}$monocytes and their DC derivatives [112]. Although the drug loading process does not seem to be cell type dependent, it remains to be determined if homing or other cellular properties are required in order to see an effect on GvHD, tumor growth or other parameters. Monocytes, DC and Treg are intrinsically capable of motility and chemotaxis, and would be predicted to localize to sites of immune interest with an efficiency equal to or greater than that of MSC, as long as their functions were not unduly inhibited by the drug cargo. While homing may be required for targeting of disseminated inflammatory foci, it is possible to see the utility of direct application of dermal fibroblasts pretreated with anti-inflammatory and/or antibiotic compounds for repair of skin, for example. There is also the potential to broaden the range of drugs that can be loaded into MSC through the use of nanoparticles embedded with other small molecules [113].

\section{Executive summary}

- In order to understand how mesenchymal stromal cells (MSC) function as therapeutic agents and to further enhance their properties, we need to address a number of basic and applied questions:

- What are the relevant immunoregulatory mechanisms deployed by MSC in vivo, and can they be licensed effectively by pre-treatments; do MSC of different origins have distinct therapeutic benefits; is it possible to increase their localization at specific sites in the body through simple pretreatments; is there selectivity or an active component to the uptake of small molecule drugs by MSC or is it primarily determined by hydrophobicity; do MSC metabolize the loaded drugs and can this be exploited to convert pro-drugs; can enhanced exosomes be derived from treated MSC; can multiple drugs be loaded without affecting MSC function; will there be reduced levels of sensitization to allogeneic MSC that have been treated with immunosuppressive drugs (ISD); is suppression of immune responses by ISD-loaded MSC temporary or can it lead to anergy or durable tolerance; what regulatory issues need to be addressed in order to use MSC loaded with standardly prescribed drugs in the clinic; what are the optimal sizes and repeats of MSC doses?

- There has been an extraordinary level of interest in exploiting MSC for a variety of clinical applications, but experience to date indicates that their acceptance as effective and economic therapeutic tools for regenerative medicine will require improvements in their production, homing, and immunoregulatory activity.

- While bone marrow has been the primary source, MSC derived from adipose and placental tissues are increasingly being used due to their ready availability and non-invasive acquisition.

- MSC may differ in their capacity to differentiate into distinct stromal cell types according to their tissue of origin, but they appear to possess similar immunoregulatory activities.

- Recent publications have reported that MSC can be significantly enhanced in their ability to mediate immune suppression and anti-cancer activities through the simple process of briefly incubating them in well-established small molecule drugs. 


\section{Future perspective}

There has been an extraordinary level of interest in exploiting MSC for a variety of clinical applications, but experience to date indicates that their acceptance as effective and economic therapeutic tools for regenerative medicine will require improvements at a number of levels: identification of the most effective MSC source; economic expansion without loss of efficacy; localization to relevant tissues; and increased potency according to the desired application. The extensive $R \& D$ and translational activity being undertaken by academic and commercial parties will undoubtedly provide advancements in all of these areas, and the recent observations of functional enhancement through simple incubations with a range of drugs provide encouragement for the development of improved formulations and potentially new applications for MSC.

\section{Open Access:}

This work is licensed under Crown copyright protection and licensed for use under the Open Government Licence unless

\section{References}

Papers of special note have been highlighted as:

- of interest

1 Dominici M, Le Blanc K, Mueller I et al. Minimal criteria for defining multipotent mesenchymal stromal cells. The International Society for Cellular Therapy position statement. Cytotherapy 8, 315-317 (2006).

2 Deans RJ, Moseley AB. Mesenchymal stem cells: biology and potential clinical uses. Exp. Hematol. 28, 875-884 (2000).

3 Kfoury Y, Scadden DT. Mesenchymal cell contributions to the stem cell niche. Cell Stem Cell 16(3), 239-253 (2015).

4 Bénézech C, Mader E, Desanti G et al. Lymphotoxin- $\beta$ receptor signaling through NF- $\mathrm{KB} 2$-RelB pathway reprograms adipocyte precursors as lymph node stromal cells. Immunity 37(4), 721-734 (2012).

5 Di Nicola M, Carlo-Stella C, Magni M et al. Human bone marrow stromal cells suppress T-lymphocyte proliferation induced by cellular or nonspecific mitogenic stimuli. Blood 99(10), 3838-3843 (2002).

6 Nauta AJ, Fibbe WE. Immunomodulatory properties of mesenchymal stromal cells. Blood 110 (10), 3499-3506 (2007).

7 Fontaine MJ, Shih H, Schäfer R, Pittenger M. Unraveling the mesenchymal stromal cells' paracrine immunomodulatory effects. Transfus. Med. Rev. 30 (1), 37-43 (2016).

8 Caplan A, Correa D. The MSC: an injury drugstore. Cell Stem Cell 9(1), 11-15 (2011).

9 Galipeau J, Krampera M, Barrett J et al. International Society for Cellular Therapy perspective on immune functional assays for mesenchymal stromal cells as potency release criterion for advanced phase clinical trials. Cytotherapy 18(2), 151-159 (2016). otherwise indicated. Where any of the Crown copyright information in this work is republished or copied to others, the source of the material must be identified and the copyright status under the Open Government Licence acknowledged.

\section{Acknowledgements}

John Girdlestone would like to thank Dr CV Navarrete at NHSBT Colindale, for support, and Saket Srivastava for personal communication of unpublished work. Apologies are offered to the many groups working on MSC whose work it was not possible to include in this review.

\section{Financial \& competing interests disclosure}

The work reported by John Girdlestone was performed in the laboratory of Dr CV Navarrete at NHSBT Colindale, supported by funding from the NIHR and Bloodwise. The author has no other relevant affiliations or financial involvement with anyorganization or entity with a financial interest in or financial conflict withthe subject matter or materials discussed in the manuscript apart from thosedisclosed. No writing assistance was utilized in the production of this manuscript.

- International Society for Cellular recommendations for characterization of immune phenotype of mesenchymal stromal cells (MSC) generated for clinical use.

10 Le Blanc K, Rasmusson I, Sundberg B et al. Treatment of severe acute graft-versus-host disease with third party haploidentical mesenchymal stem cells. Lancet 363 , 1439-1441 (2004).

- Reports efficacy of MSC in a patient with acute graftversus-host disease.

11 Karussis D, Karageorgiou C, Vaknin-Dembinsky A et al. Safety and immunological effects of mesenchymal stem cell transplantation in patients with multiple sclerosis and amyotrophic lateral sclerosis. Arch. Neurol. 67, 1187-1194 (2010).

12 García-Olmo D, García-Arranz M, Herreros D, Pascual I, Peiro C, Rodríguez-Montes JA. A Phase I clinical trial of the treatment of Crohn's fistula by adipose mesenchymal stem cell transplantation. Dis. Colon Rectum 48, 1416-23 (2005).

13 Perico N, Casiraghi F, Introna $\mathrm{M}$ et al. Autologous mesenchymal stromal cells and kidney transplantation: a pilot study of safety and clinical feasibility. Clin. J. Am. Soc. Nephrol. 6, 412-422 (2011).

14 Mangraviti A, Tzeng SY, Gullotti D et al. Non-virally engineered human adipose mesenchymal stem cells produce BMP4, target brain tumors, and extend survival. Biomaterials 100, 53-66 (2016).

15 D'souza N, Rossignoli F, Golinelli G et al. Mesenchymal stem/stromal cells as a delivery platform in cell and gene therapies. BMC Med. 13, 186 (2015).

16 Rani S, Ryan AE, Griffin MD, Ritter T. Mesenchymal stem cell-derived extracellular vesicles: toward cell-free therapeutic applications. Mol. Ther. 23(5), 812-823 (2015).

17 Zhu Y, Feng X, Abbott J et al. Human mesenchymal stem 
cell microvesicles for treatment of Escherichia coli endotoxininduced acute lung injury in mice. Stem Cells 32, 116-125 (2014))

18 Lee C, Mitsialis SA, Aslam M et al. Exosomes mediate the cytoprotective action of mesenchymal stromal cells on hypoxia-induced pulmonary hypertension. Circulation 126, 2601-2611 (2012).

19 Lee RH, Yu JM, Foskett AM et al. TSG-6 as a biomarker to predict efficacy of human mesenchymal stem/progenitor cells (hMSCs) in modulating sterile inflammation in vivo. Proc. Natl Acad. Sci. USA 111(47), 16766-16771 (2014).

O of IL-10 production by activated B cells via a cell contactdependent cyclooxygenase-2 pathway upregulated in IFN$\gamma$-treated mesenchymal stem cells. Immunobiology 221(2), 129-136 (2016).

21 Meisel R, Zibert A, Laryea M, Göbel U, Däubener W, Dilloo D. Human bone marrow stromal cells inhibit allogeneic T-cell responses by indoleamine 2,3-dioxygenase-mediated tryptophan degradation. Blood 103, 4619-4621 (2004).

22 Krampera M1, Galipeau J, Shi Y, Tarte K, Sensebe L. Immunological characterization of multipotent mesenchymal stromal cells - The International Society for Cellular Therapy (ISCT) working proposal. Cytotherapy 15(9), 1054-1061 (2013).

23 Lalu MM, McIntyre L, Pugliese C et al. Safety of cell therapy with mesenchymal stromal cells (SafeCell): a systematic review and meta-analysis of clinical trials. PLoS ONE 7(10), e47559 (2012).

- Systematic review of the clinical safety of MSC.

24 Chen X, Wang C, Yin J, Xu J, Wei J, Zhang Y. Efficacy of mesenchymal stem cell therapy for steroid-refractory acute graft-versus-host disease following allogeneic hematopoietic stem cell transplantation: a systematic review and metaanalysis. PLoS ONE 10(8), e0136991 (2015).

25 Hashmi S, Ahmed M, Murad MH et al. Survival after mesenchymal stromal cell therapy in steroid-refractory acute graft-versus-host disease: systematic review and metaanalysis. . Lancet Haematol. 3(1), e45-52 (2016).

26 Kallekleiv M, Larun L, Bruserud ø, Hatfield KJ. Cotransplantation of multipotent mesenchymal stromal cells in allogeneic hematopoietic stem cell transplantation: a systematic review and meta-analysis. Cytotherapy 18(2), 172-185 (2016).

27 Amé-Thomas P, Maby-El Hajjami H, Monvoisin C et al. Human mesenchymal stem cells isolated from bone marrow and lymphoid organs support tumor B-cell growth: role of stromal cells in follicular lymphoma pathogenesis. Blood 109(2), 693-702 (2007).

28 Krause DS, Scadden DT. A hostel for the hostile: the bone marrow niche in hematologic neoplasms. Haematologica 100(11), 1376-1387 (2015).

29 Ning H, Yang F, Jiang M et al. The correlation between cotransplantation of mesenchymal stem cells and higher recurrence rate in hematologic malignancy patients: outcome of a pilot clinical study. Leukemia 22(3), 593-599 (2008). cells for organ transplantation: different sources and unique characteristics? Curr. Opin. Organ Transplant. 19(1), 41-46 (2014).

31 Xu C, Jiang J, Sottile V, McWhir J, Lebkowski J, Carpenter MK. Immortalized fibroblast-like cells derived from human embryonic stem cells support undifferentiated cell growth. Stem Cells 22, 972-980 (2004).

32 Giuliani M, Oudrhiri N, Noman ZM et al. Human mesenchymal stem cells derived from induced pluripotent stem cells down-regulate NK-cell cytolytic machinery. Blood 118, 3254-3262 (2011).

33 Jung Y, Bauer G, Nolta JA. Concise review: induced pluripotent stem cell-derived mesenchymal stem cells: progress toward safe clinical products. Stem Cells 30(1), 42-47 (2012).

34 Pijuan-Galitó S, Tamm C, Schuster J. Human serum-derived protein removes the need for coating in defined human pluripotent stem cell culture. Nat. Commun. 7, Article number 12170 (2016).

35 Bartholomew A, Sturgeon C, Siatskas M et al. Mesenchymal stem cells suppress lymphocyte proliferation in vitro. Exp. Hematol. 30(1), 42-48 (2002).

36 Ankrum JA, Ong JF, Karp JM. Mesenchymal stem cells: immune evasive, not immune privileged. Nat. Biotechnol. 32(3), 2522-2560 (2014).

Roemeling-van Rhijn M, Reinders ME, Franquesa M. et al. Human allogeneic bone marrow and adipose tissue derived mesenchymal stromal cells induce CD8 + cytotoxic T cell reactivity. J. Stem Cell Res. Ther. 3(Suppl. 6), 004 (2013).

38 Eliopoulos N, Stagg J, Lejeune L, Pommey S, Galipeau $\mathrm{J}$. Allogeneic marrow stromal cells are immune rejected by MHC class I- and class II-mismatched recipient mice. Blood 106, 4057-4065 (2005).

39 Taylor CJ, Bolton EM, Bradley JA. Immunological considerations for embryonic and induced pluripotent stem cell banking. Philos. Trans. R. Soc. Lond. B. Biol. Sci. 366(1575), 2312-2322 (2011).

40 Mattar P, Bieback K. Comparing the immunomodulatory properties of bone marrow, adipose tissue, and birthassociated tissue mesenchymal stromal cells. Front. Immunol. 6, 560 (2015)

41 Trounson A, McDonald C. Stem cell therapies in clinical trials: progress and challenges. Cell Stem Cell 17, 11-22 (2015).

42 Wang Y, Yu X, Chen E, Li L. Liver-derived human mesenchymal stem cells: a novel therapeutic source for liver diseases. Stem Cell Res. Ther. 7(1), 71 (2016).

43 Girdlestone J, Limbani VA, Cutler AJ, Navarrete CV. Efficient expansion of mesenchymal stromal cells from umbilical cord under low serum conditions. Cytotherapy 11(6), 738-748 (2009).

44 Kern S, Eichler H, Stoeve J, Klüter H, Bieback K. Comparative analysis of mesenchymal stem cells from bone marrow, umbilical cord blood, or adipose tissue. Stem Cells 24(5), 1294-1301 (2006).

45 Reinisch A, Etchart N, Thomas D et al. Epigenetic and 
in vivo comparison of diverse MSC sources reveals an endochondral signature for human hematopoietic niche formation. Blood 125(2), 249-260 (2015).

46 Yoo KH, Jangb IK, Leea MW et al. Comparison of immunomodulatory properties of mesenchymal stem cells derived from adult human tissues. Cell. Immunol. 259(2), 150-156 (2009).

47 van der Garde M, van Pel M, Millán Rivero JE et al. Direct comparison of wharton's jelly and bone marrow-derived mesenchymal stromal cells to enhance engraftment of cord blood CD34(+) transplants. Stem Cells Dev. 24(22), 2649-2659 (2015).

48 Vangsness CT, Sternberg H, Harris L. Umbilical cord tissue offers the greatest number of harvestable mesenchymal stem cells for research and clinical application: a literature review of different harvest sites. Arthroscopy 31(9), 1836-1843 (2015).

49 Emnett RJ, Kaul A, Babic A et al. Evaluation of tissue homogenization to support the generation of GMP-compliant mesenchymal stromal cells from the umbilical cord. Stem Cells Int. 2016, 3274054 (2016).

50 Smith JR, Pfeifer K, Petry F, Powell N, Delzeit J, Weiss ML. Standardizing umbilical cord mesenchymal stromal cells for translation to clinical use: selection of GMP-compliant medium and a simplified isolation method. Stem Cells Int. 2016, 6810980 (2016).

51 Capelli C, Pedrini O, Valgardsdottir R, Da Roit F, Golay $\mathrm{J}$, Introna M. Clinical grade expansion of MSCs. Immunol. Lett.168(2), 222-227 (2015).

52 Hess DC, Sila CA, Furlan AJ, Wechsler LR, Switzer JA, Mays RW. A double-blind placebo-controlled clinical evaluation of MultiStem for the treatment of ischemic stroke. Int. J. Stroke 9(3), 381-386 (2014).

53 Kurtzberg J, Prockop S, Teira P et al. Allogeneic human mesenchymal stem cell therapy (remestemcel-L, Prochymal) as a rescue agent for severe refractory acute graft-versus-host disease in pediatric patients. Biol. Blood Marrow Transplant. 20(2), 229-235 (2014).

54 Murphy MB, Moncivais K, Caplan AI. Mesenchymal stem cells: environmentally responsive therapeutics for regenerative medicine. Exp. Mol. Med. 45, e54 (2013).

55 Cuthbert RJ, Giannoudis PV, Wang XN et al. Examining the feasibility of clinical grade CD271 +enrichment of mesenchymal stromal cells for bone regeneration. PLOS ONE 10(3), e 0117855 (2015).

56 Gronthos S, Graves SE, Ohta S, Simmons PJ. The STRO1+ fraction of adult human bone marrow contains the osteogenic precursors. Blood 84, 4164-4173 (1994).

57 Quirici N, Soligo D, Bossolasco P, Servida F, Lumini C, Deliliers GL. Isolation of bone marrow mesenchymal stem cells by anti-nerve growth factor receptor antibodies. Exp. Hematol. 30, 783-791 (2002).

58 Sacchetti B, Funari A, Michienzi S et al. Self-renewing osteoprogenitors in bone marrow sinusoids can organize a hematopoietic microenvironment. Cell 131(2), 324-336 (2007).
59 Galipeau J. The mesenchymal stromal cells dilemma - and prolong skin graft survival in vivo. Exp. Hematol. 30(1), 42-48 (2002).

60 Riordan NH, Madrigal M, Reneau J et al. Scalable efficient expansion of mesenchymal stem cells in xeno free media using commercially available reagents. J. Transl. Med. 13, 232 (2015)

61 Hemeda H, Giebel B, Wagner W. Evaluation of human platelet lysate versus fetal bovine serum for culture of mesenchymal stromal cells. Cytotherapy 16(2), 170-180 (2014).

62 Castiglia S, Mareschi K, Labanca L et al. Inactivated human platelet lysate with psoralen: a new perspective for mesenchymal stromal cell production in Good Manufacturing Practice conditions. Cytotherapy 16(6), 750-763 (2014)

63 Thirumala S, Goebel WS, Woods EJ. Manufacturing and banking of mesenchymal stem cells. Expert Opin. Biol. Ther. 13(5), 673-691 (2013).

64 Panchalingam KM, Jung S, Rosenberg L, Behie LA. Bioprocessing strategies for the large-scale production of human mesenchymal stem cells: a review. Stem Cell Res. Ther. 6, 225 (2015).

65 Salem B, Miner S, Hensel NF et al. Quantitative activation suppression assay to evaluate human bone marrow-derived mesenchymal stromal cell potency. Cytotherapy 17(12), 1675-1686 (2015).

66 Hanley PJ, Mei Z, Durett AG et al. Efficient manufacturing of therapeutic mesenchymal stromal cells with the use of the quantum cell expansion system. Cytotherapy16(8), 1048-1058 (2014).

67 Heathman TRJ, Stolzing A, Fabian Cet al. Scalability and process transfer of mesenchymal stromal cell production from monolayer to microcarrier culture using human platelet lysate. Cytotherapy 18(4), 523-535 (2016).

68 Schnitzler AC, Verma A, Kehoe DEet al. Bioprocessing of human mesenchymal stem/stromal cells for therapeutic use: current technologies and challenges. Biochem. Eng. J. 108, 3-13 (2016).

69 Gholamrezanezhad A, Mirpour S, Bagheri M et al. In vivo tracking of ${ }^{111}$ In-oxine labeled mesenchymal stem cells following infusion in patients with advanced cirrhosis. $\mathrm{Nucl}$. Med. Biol. 38(7), 961-967 (2011).

70 Leibacher J, Henschler R. Biodistribution, migration and homing of systemically applied mesenchymal stem/stromal cells. Stem Cell Res. Ther. 7(7), (2016).

71 Weiss DJ. Current status of stem cells and regenerative medicine in lung biology and diseases. Stem Cells 32(1), $16-25$ (2014).

72 Karp JM, Leng Teo GS. . Mesenchymal stem cell homing the devil is in the details. Cell Stem Cell 4(3), 206-216 (2009).

73 Ren G, Zhao X, Zhang L et al. Inflammatory cytokineinduced intercellular adhesion molecule- 1 and vascular cell adhesion molecule- 1 in mesenchymal stem cells are critical for immunosuppression. J. Immunol. 184, 2321-2328 (2010).

74 De Becker A, Riet IV. Homing and migration of 
mesenchymal stromal cells: how to improve the efficacy of cell therapy? World J. Stem Cells 8(3), 73-87 (2016). proportion of mesenchymal stem cells strongly expresses functionally active CXCR4 receptor capable of promoting migration to bone marrow. Blood 104(9), 2643-2645 (2004).

76 Bing W, Pang X, Qu Q, Bai X, Yang W, Bi Y, Bi X. Simvastatin improves the homing of BMSCs via the PI3K/AKT/miR-9 pathway. J. Cell. Mol. Med. 20(5), 949-961 (2016).

77 Li N, Yang YJ, Qian HY et al. Intravenous administration of atorvastatin-pretreated mesenchymal stem cells improves cardiac performance after acute myocardial infarction: role of CXCR4. Am. J. Transl. Res. 7(6), 1058-1070 (2015).

78 Hocking AM. The role of chemokines in mesenchymal stem cell homing to wounds. Adv. Wound Care 4(11), 623-630 (2015).

79 Kavanagh DP, Suresh S, Newsome PN, Frampton J, Kalia N. Pretreatment of mesenchymal stem cells manipulates their vasculoprotective potential while not altering their homing within the injured gut. Stem Cells 33(9), 2785-2797 (2015).

80 Khurana A, Nejadnik H, Fanny Chapelin et al. Ferumoxytol: a new, clinically applicable label for stem-cell tracking in arthritic joints with MRI. Nanomedicine 8(12), 1969-1983 (2013).

81 Sohni A, Verfaillie CM. Mesenchymal stem cells migration homing and tracking. Stem Cells Int. 2013, 130763 (2013).

82 Caplan AI. Adult mesenchymal stem cells: when, where, and how. Stem Cells Int. 2015, 628767 (2015).

83 Westenfelder C, Togel FE. Protective actions of administered mesenchymal stem cells in acute kidney injury: relevance to clinical trials. Kidney Int. Suppl. 1(3), 103-106 (2011).

84 Zanotti L, Sarukhan A, Dander E et al.

Encapsulated mesenchymal stem cells for in vivo immunomodulation. Leukemia 27, 500-503 (2013).

85 Meier RP, Mahou R, Morel P et al. Microencapsulated human mesenchymal stem cells decrease liver fibrosis in mice. J. Hepatol. 62(3), 634-641 (2015).

86 Krampera M, Cosmi L, Angeli R et al. Role for interferongamma in the immunomodulatory activity of human bone marrow mesenchymal stem cells. Stem Cells 24(2), 386-398 (2006).

87 Krampera M. Mesenchymal stromal cell 'licensing': a multistep process. Leukemia 25, 1408-1414 (2011). Pevsner-Fischer M, Morad V, Cohen-Sfady M et al. Toll-like receptors and their ligands control mesenchymal stem cell functions. Blood 109(4), 1422-1432 (2007).

89 Cho HH, Bae YC, Jung JS. Role of Toll-like receptors on human adipose-derived stromal cells. Stem Cells 24(12), 2744-2752 (2006).

90 Opitz CA, Litzenburger UM, Lutz C et al. Toll-like receptor engagement enhances the immunosuppressive properties of human bone marrow-derived mesenchymal stem cells by inducing indoleamine-2,3-dioxygenase-1 via interferon-b and protein kinase R. Stem Cells 27, 909-919 (2009).
91 DelaRosa O, Dalemans W, Lombardo E. Toll-like receptors as modulators of mesenchymal stem cells. Front. Immunol. 3, 182 (2012).

92 Waterman RS, Tomchuck SL, Henkle SL, Betancourt AM. A new mesenchymal stem cell (MSC) paradigm: polarization into a pro-inflammatory MSC1 or an immunosuppressive MSC2 phenotype. PLoS ONE 5(4), e10088 (2010).

93 Waterman RS, Henkle SL, Betancourt AM. Mesenchymal stem cell 1 (MSC1)-based therapy attenuates tumor growth whereas MSC2-treatment promotes tumor growth and metastasis. PLoS ONE 7(9), e45590 (2012).

94 Cutler AJ, Limbani V, Girdlestone J, Navarrete CV. Umbilical cord-derived mesenchymal stromal cells modulate monocyte function to suppress $\mathrm{T}$ cell proliferation. J. Immunol. 185(11), 6617-6623 (2010).

95 Girdlestone J, Pido-Lopez J, Srivastava S et al. Enhancement of the immunoregulatory potency of mesenchymal stromal cells by treatment with immunosuppressive drugs. Cytotherapy 17(9), 1188-1199 (2015).

- Demonstrates that rapamycin-treated MSC are effective in a preclinical model of graft-versus-host disease.

96 Hoogduijn MJ, Crop MJ, Korevaar SS et al. Susceptibility of human mesenchymal stem cells to tacrolimus, mycophenolic acid, and rapamycin. Transplantation 86(9), 1283-1291 (2008).

97 Trepanier DJ, Gallant H, Legatt DF, Yatscoff RW. Rapamycin: distribution, pharmacokinetics and therapeutic range investigations: an update. Clin. Biochem. 31, 345-351 (1998).

98 Moes DJ, Guchelaar H-J, de Fijter JW. Sirolimus and everolimus in kidney transplantation. Drug Discov. Today 20(10), 1243-1249 (2015).

99 Orange DE, Blachere NE, Fak J et al. Dendritic cells loaded with FK506 kill $\mathrm{T}$ cells in an antigen-specific manner and prevent autoimmunity in vivo. eLife2, e00105 (2013).

100 Singh K, Kozyr N, Stempora L et al. Regulatory T cells exhibit decreased proliferation but enhanced suppression after pulsing with sirolimus. Am. J. Transplant. 12, 1441-1457 (2012).

101 Kim K-W, Moon S-J, Park M-J et al. Optimization of adipose tissue-derived mesenchymal stem cells by rapamycin in a murine model of acute graft-versus-host disease. Stem Cell Res.Ther. 6, 202 (2015).

102 Ge W, Jiang J, Baroja ML et al. Infusion of mesenchymal stem cells and rapamycin synergize to attenuate alloimmune responses and promote cardiac allograft tolerance. . Am. J. Transplant. 9, 1760-1772 (2009).

103 Cheng P-P, Liu X-C, Ma P-F et al. iPSC-MSCs combined with low-dose rapamycin induced islet allograft tolerance through suppressing Th1 and enhancing regulatory T-cell differentiation. Stem Cells Dev. 24(15), 1793-1804 (2015).

104 Togha M, Jahanshahi M, Alizadeh L et al. Rapamycin augments immunomodulatory properties of bone marrow-derived mesenchymal stem cells in experimental autoimmune encephalomyelitis. Mol. Neurobiol. doi:10.1007/s12035-016-9840-3 (2016) (Epub ahead of print). 
105 Dean PG, Lund WJ, Larson TS et al. Woundhealing complications after kidney transplantation: a prospective, randomized comparison of sirolimus and tacrolimus. Transplantation 77(10), 1555-1561 (2004).

106 Pessina A, Bonomi A, Coccè V et al. Mesenchymal stromal cells primed with paclitaxel provide a new approach for cancer therapy. PLoS ONE 6(12), e28321 (2011).

- $\quad$ MSC are shown to exhibit anticancer effects after loading with paclitaxel.

107 Bonomi A, Sordi V, Dugnani E et al. Gemcitabine-releasing mesenchymal stromal cells. Cytotherapy 17(12), 1687-1695 (2015).

108 Sisto F, Bonomi A, Cavicchini L et al. Human mesenchymal stromal cells can uptake and release ciprofloxacin, acquiring in vitro anti-bacterial activity. Cytotherapy 16(2), 181-190 (2014).

109 Conforti A, Biagini S, Starc N et al. Human mesenchymal stromal cells primed with paclitaxel, apart from displaying anti-tumor activity, maintain their immune regulatory functions in vitro. Cytotherapy 16(6), 868-870 (2014).

110 Pessina A, Leonetti C, Artuso S et al. Drug-releasing mesenchymal cells strongly suppress B16 lung metastasis in a syngeneic murine model. J. Exp. Clin. Cancer Res. 34, 82 (2015).

111 Coccè V, Vitale A, Colombo $\mathrm{S}$ et al. Human skin-derived fibroblasts used as a 'Trojan horse' for drug delivery. Clin. Exp. Dermatol. 41(4), 417-424 (2016).

112 Bonomi A, Lisini D, Navone SE et al. Human CD14+ cells loaded with paclitaxel acquiring in vitro anti-bacterial activity. Cytotherapy 16(2), 181-190 (2014).

113 Sarkar D, Ankrum JA, Teo GSL, Carman CV, Karp JM. Cellular and extracellular programming of cell fate through engineered intracrine-, paracrine-, and endocrine-like mechanisms. Biomaterials 32(11), 3053-3061 (2011). 